\section{CHOLEDOCHUS CYST}

\author{
BY
}

\author{
S. H. TAYLOR, M.B., Ch.B., B.Sc. \\ Formerly House-Physician, Department of Medicine, \\ Queen Elizabeth Hospital, Birmingham \\ AND
}

\section{G. SLANEY, M.B., F.R.C.S.}

Surgical Registrar, Department of Surgery, Queen Elizabeth Hospital, Birmingham

Choledochus cyst is a rare cause of jaundice in which a part or the whole of the extrahepatic biliary tract shows gross dilatation, usually of a saccular type. The anatomy of this congenital anomaly was first described by Vater in 1723, and fewer than 200 cases have since been reported (Gross, 1933; Walton, 1939; Shallow, Eger, and Wagner, 1946). Sir James Walton was one of the few authors to describe more than two cases of his own, and he states that these were the only two seen among 23,048 necropsies at the London Hospital in the 32 years prior to 1938 . Likewise, Judd and Greene (1928) found only one case of choledochal cyst in 17,381 operations on the biliary tract.

Most authors believe the lesion arises from a congenital weakness of a localized portion of the wall of the common bile or hepatic ducts, this defect probably occurring at the site of a diverticulum or an incompletely developed accessory hepatic duct. Moreover, once the cyst attains a certain size a vicious circle is created, for drainage into the last portion of the common bile duct is further impeded by the pressure of the eccentrically lying cyst.

Pressure of the cyst on surrounding structures, including the extrahepatic biliary system, accounts for much of the symptomatology of the condition. Although the cyst may vary greatly in size, the majority are $10-15 \mathrm{~cm}$. in diameter and usually contain bile, although sometimes the fluid is clear. Gall-stones may be present, and in a case described by Irwin and Morison (1945) a squamous-celled carcinoma was found in the wall of the sac. The dilatation of the common bile duct often extends into the common hepatic duct, but the gallbladder and cystic duct are usually of normal size. The terminal portion of the common bile duct is often narrowed, and sometimes cannot be found even when obstruction is incomplete (Walton, 1939).

\section{The Clinical Syndrome}

The lesion occurs predominantly in females, and symptoms frequently arise between the ages of 5 and 25 years. Shallow, Eger, and Wagner (1943) found that $75 \%$ of cases described in the literature were diagnosed before 25 years of age, and Lichtman (1949) reports that $80 \%$ of cases have occurred in females. Hertzler and Maguire (1951) state that in $80 \%$ of the reported cases congenital cysts of the common duct occur in patients under 25 years of age and $60 \%$ of cases occur in children under the age of 10 years. The most usual presenting symptoms are recurrent jaundice, upper abdominal pain which often resembles biliary colic, and a tumour in the right hypochondrium. Pregnancy has often appeared to be a precipitating factor in the onset of these symptoms. In many cases in which the cyst was not palpable the clinical syndrome was identical with that of gall-stone obstruction of the biliary tract, this being the diagnosis most often made. Cholecystectomy has even been performed, the true diagnosis and the presences of the cyst being missed at laparotomy. Neoplasm is seldom considered on account of the youth of many of the patients.

Demonstration of the cyst directly by cholecystography is usually not possible, but the gall-bladder is often distorted so that it appears comma-like. Calcification may occur in the cyst wall, so that its presence may be confirmed on a plain radiograph of the abdomen, and distortion of the duodenum or colon may on occasion be shown by barium studies.

Few studies have been reported in patients with this condition owing to the infrequency with which the diagnosis was made pre-operatively. The following case is of interest because of the typical features of the clinical syndrome as it presented and the studies that were made possible by the pre-operative diagnosis.

\section{Case Report}

A married woman aged 19 had suffered five intermittent attacks of mild jaundice, each lasting a few days, during the two years before admission; these had been unassociated with abdominal pain or any symptoms referable to the alimentary tract. The attack of jaundice which precipitated her admission to a maternity hospital began three months previously, during the sixth month of her first pregnancy. Following a premature delivery at the seventh month, the jaundice largely resolved, but increased again ten days later after the patient had returned home. She was readmitted to hospital deeply icteric and suffering from a mild diarrhoea with three or four pale semi-formed stools daily. A mass in the right hypochondrium, at this time thought to be the liver, was palpable. Cholecystography revealed no abnormality of the gall-bladder, and liver-function tests showed a predominantly obstructive pattern.

She was transferred to the medical unit, Queen Elizabeth Hospital, one week later, on March 4, 1954. She was slightly icteric and rather pale, but there were no abnormal physical signs other than a firm rounded tumour in the right hypochondrium, about $10 \mathrm{~cm}$. in diameter. The liver and spleen were not palpable and a diagnosis of choledochus cyst seemed to be justified. Laboratory investigations showed a normal blood count, but the erythrocyte sedimentation rate was $45 \mathrm{~mm}$. in one hour (Wintrobe corrected). Radiography of the chest and soft abdominal tissues showed nothing abnormal, but barium studies demonstrated a narrowing of the second part of the duodenum with dilatation of its proximal portion. There was a moderate excess of fattyacid crystals in the stools, and fat-balance studies on a daily intake of $50 \mathrm{~g}$. of fat revealed a moderately severe degree of steatorrhoea with only $72 \%$ absorption of ingested fat. Occult blood was not found in the stools. Liver-function tests were normal except for a raised serum bilirubin (5 mg./100 ml.), a raised serum alkaline phosphatase (29 units $/ 100 \mathrm{ml}$. ), and a prothrombin value of $48 \%$ by Quick's method. The urine contained urobilin, bilirubin, and bile salts in detectable quantities.

At operation a large ovoid cyst was found arising from the common bile duct. It measured 20 by $10 \mathrm{~cm}$. and contained about one litre of dark green bile. The gall-bladder and cystic duct were normal. The cyst was adherent to many of the surrounding structures, prohibiting its complete resection. It was therefore drained into the jejunum by means of a Roux-en-Y anastomosis.

The patient made a satisfactory post-operative recovery until the 11th post-operative day, when she developed diarrhoea with three to six stools a day. The stools were semi-fluid, pale, rather bulky, and offensive, and fat-balance studies again showed a severe degree of steatorrhoea with only $64 \%$ absorption of ingested fat. This fatty diarrhoea continued up to the time of discharge on the 37th day after operation. During the five weeks following operation the biochemical liver-function tests remained normal, and although the serum bilirubin and prothrombin values gradually returned to normal the serum alkaline phosphatase remained persistently elevated at $30-50$ units $/ 100 \mathrm{ml}$. 
Intestinal intubation showed the duodenal contents contained normal amounts of pancreatic enzymes, but there was a complete absence of bile from the small intestine as far.as the jejunum. It appeared likely, therefore, that the anastomosis between the cyst and the small intestine was not sufficiently proximal to ensure adequate mixing of the pancreatic enzymes and their biliary activators during the processes of digestion. However, before further surgery could be carried out the patient requested discharge, and since that time has refused review. During her stay in hospital the patient showed no significant weight loss, haematological change, or any other clinical manifestations of a general malabsorption syndrome.

\section{Discussion}

This patient had many of the clinical features usually associated with this anomaly. She was a young woman with recurrent attacks of mild jaundice and a tumour in the right hypochondrium. The most severe attack of jaundice was associated with pregnancy and resolved following delivery. Laboratory investigations were helpful diagnostically only in so far as they confirmed the presence of an obstructive biliary lesion, and contrast radiography was of value in excluding a tumour of intestinal origin. The treatment of congenital cystic dilatation of the common bile duct is surgical, and there is no doubt that the most satisfactory procedure is choledochojejunostomy using a Roux-en-Y loop. Choledochoduodenostomy has often given satisfactory results initially, but ascending cholangitis and hepatitis have been a frequent sequel to this type of operation.

Of particular interest in the present case was the unexplained steatorrhoea observed before operation, and there is no report in the literature of this abnormality in association with choledochus cyst. A possible explanation is that distension of the upper gastro-intestinal tract with food caused intermittent but complete occlusion of the biliary tract by displacement of the cyst, so that the biliary activators of the pancreatic enzymes were absent at the most crucial phase of digestion in the upper intestine. At other times during the post-absorptive phase, biliary flow was relatively free. A further possibility is that the two conditions are entirely unrelated, the steatorrhoea being due to an idiopathic malabsorption syndrome. Although this latter possibility cannot be entirely ruled out, it is unlikely in view of the normal intestinal mucosal pattern seen on the bariummeal examination and the absence of haematological abnormality.

In spite of the relief of the obstructive element in the syndrome, the steatorrhoea continued unchanged and after operation the patient was excreting $36 \%$ of her total ingested fat. The pancreatic secretions were known to be normal in quantity and activity, and it is possible that this malabsorption was due to defective mixing of the food with the pancreatic enzymes and bile.

\section{Summary}

A case of choledochus cyst is described. Metabolic studies, with particular reference to fat absorption, were made before and after operation. The pre-operative steatorrhoea persisted after an operation which relieved the biliary obstruction.

We thank Professor W. Melville Arnott and Mr. B. N. Brooke, under whose care this patient was admitted, for their help and encouragement in the preparation of this paper. We also thank Dr. C. F. J. Cropper, of Worcester Royal Infirmary, who referred this case initially, and Mr. Garfield Thomas, who was responsible for the biochemical investigations.

\section{REFERENCES}

Gross, R. E. (1933). J. Pediat., 3, 730. Irwin, S. T., and Morison. J. E. (1945). Brit. J. Surg., 32, 319.

Judd, E. S. and Greene, E. I. (1928). Surg. Gynec. Obstet. 46, 317. Jichtman, S. S. (1949). Diseases of the Liver, Gall Bladder, and Bile Duct, 2nd ed. Kimpton, London.

2nd ed. K., Eger, S. A., and Wagner, F. B. (1943). Ann. Surg., 117, 355 .

Walton, Sir J. (1939). Brit. J. Surg., $27,295$.

\section{Medical Memoranda}

\section{Bilateral Rupture of Rectus Femoris Tendons in Chronic Nephritis}

The following case is rare enough to warrant recording, particularly in view of the young age of the patient and the associated general disease. Rupture of both quadriceps tendons has been recorded before, but these cases have been in elderly people (Lloyd, 1949).

\section{Case Report}

A man of 34 was admitted to hospital on March 17, 1956, having fallen on to his knees while going down some steps. On attempting to stand his knees gave way. He stated that for some months he had been feeling rather tired and that this came on after a bout of influenza. He had, however, been able to carry on his work.

On examination he was rather pale, with a blood pressure of $160 / 80 \mathrm{~mm}$. Hg. He was almost completely bald, and this made him appear much older than his years. Both knees were swollen and clinically resembled a haemarthrosis. He was very tender over the upper margin of both patellae, but there was no palpable defect in the extensor apparatus. He was just able to maintain full extension, but this caused him considerable pain. He had sustained an injury to the left ankle which proved to be a first-degree external rotation fracture of the fibula. $X$-ray examination of the knees showed that both the upper margins of the patellae were tilted forward and there was a calcified plaque in the soft tissues immediately above. It was thought that there was a tear of the rectus femoris tendon alone without involvement of the lateral expansions, and exploration of the knees was advised.

Operation.-The lesion was found to be almost identical on both sides, that on the right being slightly more extensive. A 2-in. $(5-\mathrm{cm}$.) crescent of rectus femoris tendon had been torn off the top of the patella along with a sliver of bone. There was no evidence of calcification in the tendon itself. The lateral expansions were intact, the tear being much less extensive than in the usual quadriceps rupture. The patellae were displaced forward at the site of injury and the rectus femoris tendons tucked in behind. A repair was easily accomplished with silk mattress sutures.

The patient was rather lethargic after operation, but his condition did not cause any concern until the third day, when it became obvious that there had been a sudden serious deterioration in his condition. Investigation showed : blood urea, $312 \mathrm{mg}$. per $100 \mathrm{ml}$.; $\mathrm{CO}_{2}$-combining power, 11.2 $\mathrm{mEq} / \mathrm{l}$. ; Hb, $50 \%$; serum phosphate, $12.2 \mathrm{mg}$. per $100 \mathrm{ml}$. $\mathrm{He}$ was passing normal quantities of urine, the specific gravity of which was 1010, with an albumin estimation of 70 mg. per $100 \mathrm{ml}$. On going into his previous history it was discovered that he had been rejected by the Army during the war because of albuminuria. He was regarded as a case of chronic nephritis, probably in the terminal phase. $\mathrm{He}$ shortly developed a pericarditis and died in uraemic convulsions on March 26.

Post-mortem Examination.-Both kidneys were found to be small and sclerotic. Sections of the rectus femoris tendons at the site of rupture showed no evidence of calcification or of degenerative changes.

\section{COMMENT}

It is felt that simultaneous rupture of both rectus femoris tendons in so young a patient must be an almost unique occurrence. The severe debility from his renal failure may have been a contributory cause, although there was no evidence of degenerative changes in the tendons. It is interesting to note the very limited extent of the lesion, distinguishing it from the more common form of quadriceps rupture. In the latter injury it is most unusual for the 